\title{
Data Assimilation Using the Global Ionosphere-Thermosphere Model ${ }^{\star}$
}

\author{
I.S. Kim ${ }^{1}$, J. Chandrasekar ${ }^{1}$, A. Ridley ${ }^{2}$, and D.S. Bernstein ${ }^{1}$ \\ ${ }^{1}$ Department of Aerospace Engineering, University of Michigan, Ann Arbor \\ dsbaero@umich.edu \\ ${ }^{2}$ Department of Atmospheric, Oceanic and Space Sciences, \\ University of Michigan, Ann Arbor
}

\begin{abstract}
We consider a data assimilation technique for coupled ionospheric and thermospheric dynamics. The Global Ionosphere-Thermosphere Model (GITM) is used to simulate the ionospheric and thermospheric dynamics, and evaluate the performance of the data assimilation scheme that estimates the ion densities and flow speeds. This estimation technique is based on the state dependent Riccati equation (SDRE), which uses a frozen linear dynamics matrix for the time update of the error covariance and the evaluation of the Kalman filter gain. We demonstrate the performance of the data assimilation technique on a section of the ionosphere.
\end{abstract}

\section{Data Assimilation for Space Weather Prediction}

The Sun drives our atmosphere in many ways. The most commonly understood way is through the heating of the lower atmosphere where sunlight heats the atmosphere causing weather. A similar process occurs in the thermosphere (above about 60 miles), where the thin atmosphere absorbs sunlight, and the atmosphere is heated dramatically. Another way in which the Sun affects the upper atmosphere is through the transfer of electromagnetic energy. The Sun's atmosphere flows away from it at supersonic speeds and encounters the Earth's magnetic field, transferring a significant amount of electromagnetic energy to our magnetosphere. This energy flows throughout the magnetosphere until it either leaves the system or is deposited into the upper atmosphere. This energy takes the form of strong electromagnetic currents and aurora, that is, the Northern and Southern lights.

During quiet times, when the Sun's atmosphere is calm, the heating of the atmosphere through sunlight is the dominant process. But the Sun sometimes erupts, sending large amounts of plasma and strong magnetic fields toward the Earth in events called coronal mass ejections (CMEs). When CMEs reach the Earth, they cause the Earth's magnetosphere to shrink significantly, bringing the aurora to much lower latitudes (sometimes over the United States). During these

\footnotetext{
* This research was supported by the National Science Foundation under grants CNS0539053 and ATM-0325332.
} 
disturbed periods, the electromagnetic energy injected into the atmosphere can become the dominant process, driving the atmosphere in ways that we do not fully comprehend.

In 1989, the entire province of Quebec, Canada, experienced a power blackout for 9 hours as a result of a CME. More recently, in October 2003, the so-called "Halloween storms" caused major distortions of the Earth's radiation belt and magnetic field, disrupting radio communication systems. These types of solar storms are known or suspected to have damaged several satellites in orbit around the Earth, in particular, an AT\&T Telestar 401 satellite in 1997, a PanAmSat Galaxy IV satellite in 1998, and a Kodama communications satellite in 2003 (as a result of the Halloween storms). One of the primary mechanisms for damage, especially in the electric grid, is fluctuations in the magnetic field, which induce currents through Faraday's law.

Solar storms also degrade the accuracy of the Global Positioning System, which is used for military and civilian positioning systems. This degradation occurs because the GPS signals travel through the ionosphere, which modifies their travel time from the satellite to the receiver. If there are strong changes in the ion density in the ionosphere, then the normal correction for the ionosphere is no longer accurate, causing errors in position. While this navigation error might not matter to the typical automobile, it has significant ramifications for aircraft during landings in poor visibility as well as for GPS-guided missiles.

Modeling and prediction of the upper atmosphere and the near-Earth space environment is generally referred to as space weather. This relatively new field is challenging because of the limited number of measurements in space. However, the situation has started to improve with increasing awareness of the serious effects that space weather has on terrestrial and space-based systems.

The present paper describes a Kalman filter for data assimilation based on a coupled model of the thermosphere and ionosphere. For this purpose we use the GITM model [5], which contains various species of both ions and neutrals, as well as temperatures and winds for both ions and neutrals. The domain of the model extends from $100 \mathrm{~km}$ to approximately $600 \mathrm{~km}$ altitude over the entire globe in latitude and longitude. We present preliminary data assimilation results based on a one-dimensional (vertical) model of the atmosphere. Future work will involve extensions to 3-dimensional data assimilation, assimilation of data from incoherent scatter radar, and techniques for using the data assimilation results to redirect the radar during real-time operation, that is, targeted data assimilation.

\section{Kalman Filtering for GITM}

Classical Kalman filtering is based on the linear dynamics and measurements

$$
\begin{gathered}
x_{k+1}=A_{k} x_{k}+B_{k} u_{k}+w_{k} \\
y_{k}=C_{k} x_{k}+v_{k} .
\end{gathered}
$$


The objective is to use the measurements $y_{k}$ to estimate unmeasured state components. The forecast step consists of the simulation and measurement step

$$
x_{k+1}^{\mathrm{f}}=A_{k} x_{k}^{\mathrm{da}}+B_{k} u_{k}, \quad y_{k}^{\mathrm{f}}=C_{k} x_{k}^{\mathrm{f}},
$$

while the data assimilation step is given by the "closed-loop" data-driven update

$$
x_{k}^{\mathrm{da}}=x_{k}^{\mathrm{f}}+K_{k}\left(y_{k}-y_{k}^{\mathrm{f}}\right) .
$$

Here, $x_{k}^{\mathrm{f}}$ and $x_{k}^{\mathrm{da}}$ are the forecast estimate and the data assimilation estimate. The data-injection gain $K_{k}$ involves the error covariance matrices updated by

$$
\begin{gathered}
P_{k}^{\mathrm{da}}=\left(I-K_{k} C_{k}\right) P_{k}^{\mathrm{f}}, \quad P_{k+1}^{\mathrm{f}}=A_{k} P_{k}^{\mathrm{da}} A_{k}^{\mathrm{T}}+Q_{k}, \\
K_{k}=P_{k}^{\mathrm{f}} C_{k}^{\mathrm{T}}\left(R_{k}+C_{k} P_{k}^{\mathrm{f}} C_{k}^{\mathrm{T}}\right)^{-1} .
\end{gathered}
$$

Here, $Q_{k}$ and $R_{k}$ are the covariances of $w_{k}$ and $v_{k}$, respectively.

Since GITM is nonlinear, nonlinear estimation algorithms are needed. One approach is the extended Kalman filter (XKF) in which the nonlinear dynamics advance the estimate, while the Jacobian of the dynamics propagates the covariance. Alternatively, the ensemble Kalman filter (EnKF) 4, 3] is often used. The size of the ensemble needed to obtain accurate estimates is crucial [4.

An alternative approach is the "frozen-linear" state-dependent Riccati equation approach, wherein the nonlinear dynamics advance the estimate, while an exact, state-dependent dynamics matrix propagates the pseudo covariance [2. Hence, we rewrite the nonlinear dynamics

$$
x_{k+1}=f\left(x_{k}, k\right)+B\left(x_{k}\right) u_{k}
$$

in the factored form

$$
x_{k+1}=A\left(x_{k}, k\right) x_{k}+B\left(x_{k}\right) u_{k} .
$$

This factorization is not unique, and there do not exist general principles for constructing favorable factorizations [1]. However, since no Jacobian exists for finite volume schemes with nondifferentiable limiters, the use of an SDRE factorization seems appropriate. Stability is discussed in 2]. For the system considered in the present paper, we use an SDRE factorization whose performance is similar to the performance of an extended Kalman filter in which the points of discontinuity are ignored.

\section{Model Description}

GITM models the thermosphere and ionosphere using a stretched 3D spherical grid in latitude and altitude. The number of grid points can be specified, so that the resolution is extremely flexible. GITM solves for the neutral densities of $\mathrm{O}, \mathrm{O}_{2}, \mathrm{~N}\left({ }^{2} \mathrm{D}\right), \mathrm{N}\left({ }^{2} \mathrm{P}\right), \mathrm{N}\left({ }^{4} \mathrm{~S}\right), \mathrm{N}_{2}, \mathrm{NO}, \mathrm{H}$, and $\mathrm{He}$; and ion species $\mathrm{O}^{+}\left({ }^{4} \mathrm{~S}\right)$, $\mathrm{O}^{+}\left({ }^{2} \mathrm{D}\right), \mathrm{O}^{+}\left({ }^{2} \mathrm{P}\right), \mathrm{O}_{2}^{+}, \mathrm{N}^{+}, \mathrm{N}_{2}^{+}, \mathrm{NO}^{+}, \mathrm{H}^{+}$, and $\mathrm{He}^{+}$. By not assuming hydrostatic equilibrium, the vertical solver is different from other thermospheric codes. Furthermore, GITM can simulate above a particular location on the earth (onedimensional) or a spherical section around the earth (three-dimensional). Here, we simulate a one-dimensional grid, and ignore horizontal transport but retain 
vertical advection. Three-dimensional source terms are included, which includes processes such as ion drag.

\subsection{Neutral Dynamics}

In the thermosphere, the neutrals are treated as having individual vertical velocities $\mathbf{u}_{s}$, where $s$ denotes the species. The mass density is the sum of the species densities

$$
\rho=\sum_{s} M_{s} N_{s}
$$

where $M_{s}$ is the molecular mass and $N_{s}$ is the number density of species $s$. In the vertical (or radial) direction, the natural logarithm of the total mass density $\rho$ and number densities $N_{s}$ are used as the primitive variables

$$
\mathcal{R} \triangleq \ln (\rho), \quad \mathcal{N}_{s} \triangleq \ln \left(N_{s}\right)
$$

and the continuity equation for each neutral species is

$$
\frac{\partial \mathcal{N}_{s}}{\partial t}+\nabla \cdot \mathbf{u}_{s}+\mathbf{u}_{s} \cdot \nabla \mathcal{N}_{s}=S_{N}
$$

where the source $S_{N}$ includes eddy diffusion and chemical sources and losses.

Next, we define the normalized temperature

$$
\mathcal{T} \triangleq p / \rho
$$

with $p$ the total neutral pressure. The momentum equations for species $s$ are

$$
\frac{\partial \mathbf{u}_{s}}{\partial t}+\mathbf{u}_{s} \cdot \nabla \mathbf{u}_{s}+\frac{k}{M_{s}} \nabla T+\frac{k}{M_{s}} T \nabla \mathcal{N}_{s}=S_{u},
$$

where $T \triangleq \frac{m_{n}}{k} \mathcal{T}, \bar{m}_{n}$ is the number density weighted average mass, and the source term $S_{u}$ includes friction between species, gravitational, centrifugal, and Coriolis forces. For numerical accuracy, the energy equation is expressed as the normalized temperature $\mathcal{T}$ instead of the exponentially varying $p$ as in

$$
\frac{\partial \mathcal{T}}{\partial t}+\mathbf{u} \cdot \nabla \mathcal{T}+(\gamma-1) \mathcal{T} \nabla \cdot \mathbf{u}=S_{T},
$$

where

$$
\mathbf{u} \triangleq \frac{1}{\rho} \sum_{s} M_{s} N_{s} \mathbf{u}_{s}
$$

$\gamma=5 / 3$ is the ratio of specific heats and the source term $S_{T}$ includes solar extreme ultraviolet heating and cooling terms as described in [6].

\subsection{Ion Advection}

Ion continuity equation in the vertical direction is given by

$$
\frac{\partial \mathcal{N}_{j}}{\partial t}+v_{r} \frac{\partial \mathcal{N}_{j}}{r}=S_{j}
$$

where $\mathcal{N}_{j}$ is the $\log$ of the number density of the ion species $j$ and $v_{r}$ is ion velocity. The source $S_{j}$ is obtained from the chemistry between ions and neutrals. 
We specify ions for advection using (8), while other ion densities are controlled by reactions [5] set by $\frac{\partial \mathcal{N}_{j}}{r}=0 . \mathrm{O}^{+}$is the dominant ion in the low collision region of the ionosphere, and the most important species to advect [7].

\section{Numerical Schemes}

We describe the numerical scheme used in GITM for solving the advection equations and obtain a parametrization $A\left(x_{k}, k\right)$ for SDRE-based data assimilation.

\subsection{Spatial Discretization for Advection}

We perform data assimilation on the ion species, which has greater number density variation than the neutral species. That is, although we advect both the neutral and ion species, we use measurements to directly update the estimates of only the ion species. We describe the numerical scheme used to advect the ion species as in [5]. Note (8) can be expressed as

$$
\frac{\partial U}{\partial t}+T \frac{\partial}{\partial r} U=S
$$

where $U \in \mathbb{R}^{10}$ is defined by

$$
U \triangleq\left[\mathcal{N}_{O^{+}\left({ }^{4} S\right)} \mathcal{N}_{\mathrm{O}_{2}^{+}} \mathcal{N}_{N_{2}^{+}} \mathcal{N}_{N^{+}} \mathcal{N}_{\mathrm{NO}^{+}} \mathcal{N}_{\mathrm{O}^{+}\left({ }^{2} D\right)} \mathcal{N}_{\mathrm{O}^{+}\left({ }^{2} P\right)} \mathcal{N}_{\mathrm{H}^{+}} \mathcal{N}_{\mathrm{He}} \mathrm{v}_{r}\right]^{\mathrm{T}},
$$

$T \in \mathbb{R}^{10 \times 10}$ is defined by

$$
T \triangleq \operatorname{diag}\left(v_{r}, 0, \ldots, 0\right),
$$

and for $i=1, \ldots, 10$, the $i$ th entry of $S \in \mathbb{R}^{10}$ denotes the source for the $i$ th ion species. We advect only $O^{+}\left({ }^{4} S\right)$; other states in $U$ are updated using only source terms. It follows from [5] that the time update is given by

$$
U_{j}(k+1)=U_{j}(k)-\frac{t_{s}(k)}{\Delta r_{j}}\left[T_{j}(k)\left(U_{j+\frac{1}{2}}(k)-U_{j-\frac{1}{2}}(k)\right)-\left(F_{j+\frac{1}{2}}-F_{j-\frac{1}{2}}\right)\right]+S_{j}(k)
$$

where $U_{j}$ denotes $U$ at the center of the cell $j$ and $U_{j+\frac{1}{2}}$ denotes $U$ at the edge between cells $j$ and $j+1$. For all $j=3, \ldots, n-2$, we evaluate $U_{j+\frac{1}{2}}$ by using the modified monotized central limiter [5]. Also, $F_{j+\frac{1}{2}}$ is the flux at the edge between cells $j$ and $j+1$ evaluated using the Lax-Friedrichs 2nd-order scheme.

The boundary conditions for the one-dimensional GITM model are $U_{1}(k)$, $U_{2}(k), U_{n-1}(k)$, and $U_{n}(k)$. Hence, it follows from (12) that the dynamics are

$$
x_{k+1}=f\left(x_{k}, u_{k}\right)
$$

where the state vector $x_{k} \in \mathbb{R}^{10(n-4)}$ is defined by

$$
x_{k} \triangleq\left[U_{3}(k)^{\mathrm{T}} \cdots U_{n-2}(k)^{\mathrm{T}}\right]^{\mathrm{T}}
$$

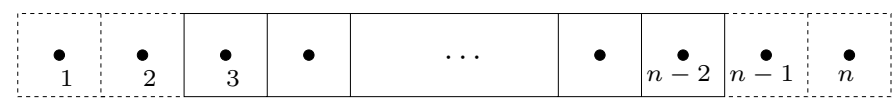

Fig. 1. One-dimensional grid used in the finite volume scheme 
and $u_{k} \in \mathbb{R}^{10(4)+10(n-4)}$ is defined by

$$
u_{k} \triangleq\left[U_{1}(k)^{\mathrm{T}} U_{2}(k)^{\mathrm{T}} U_{n-1}(k)^{\mathrm{T}} U_{n}(k)^{\mathrm{T}} S_{3}(k)^{\mathrm{T}} \cdots S_{n-2}(k)^{\mathrm{T}}\right]^{\mathrm{T}} .
$$

\subsection{State-Dependent Frozen-Linear Representation}

To express the update equation (13) as a state-dependent frozen-linear discretetime equation, we write (12) as

$$
U_{j}(k+1)=\mathcal{A}_{L 2}^{[j]} U_{j-2}(k)+\mathcal{A}_{L 1}^{[j]} U_{j-1}(k)+\mathcal{A}_{M}^{[j]} U_{j}(k)+\mathcal{A}_{R 1}^{[j]} U_{j+1}(k)+\mathcal{A}_{R 2}^{[j]} U_{j+2}(k)+S_{j}(k)
$$

where $\mathcal{A}_{L 2}^{[j]}, \mathcal{A}_{L 1}^{[j]}, \mathcal{A}_{M}^{[j]}, \mathcal{A}_{R 1}^{[j]}, \mathcal{A}_{R 2}^{[j]} \in \mathbb{R}^{10 \times 10}$ depend on $U_{i}(k), i=j-2, \ldots, j+2$, e.g.,

$$
\mathcal{A}_{j, L 2}=-\frac{t_{s}(k)}{\Delta r_{j}}\left\{-\frac{1}{4} T_{j}(k) K_{j-1}^{L}-\frac{1}{4} \max \left(c_{j-\frac{3}{2}}, c_{j-\frac{1}{2}}\right) K_{j-1}^{L}\right\},
$$

where $K_{j}^{L}$ depends on the limiter [1] and $c_{j+\frac{1}{2}}$ is the maximum wave speed at the right edge of the $j$ th cell [5].

Hence, it follows from (12) and (16) that

$$
x_{k+1}=\mathcal{A}\left(x_{k}\right) x_{k}+\tilde{u}_{k},
$$

where $\tilde{u}_{k}$ represents source terms and boundary conditions, and the block pentadiagonal matrix $\mathcal{A} \in \mathbb{R}^{10(n-4) \times 10(n-4)}$ is defined by

$$
\mathcal{A}\left(x_{k}\right) \triangleq\left[\begin{array}{ccccccc}
\mathcal{A}_{\mathrm{M}}^{[3]} & \mathcal{A}_{\mathrm{R} 2}^{[3]} & \mathcal{A}_{\mathrm{R} 2}^{[3]} & 0 & 0 & \ldots & 0 \\
\mathcal{A}_{\mathrm{L}]}^{[4]} & \mathcal{A}_{\mathrm{M}}^{[4]} & \mathcal{A}_{\mathrm{R}}^{4]} & \mathcal{A}_{\mathrm{R} 2}^{[4]} & 0 & \ldots & 0 \\
\mathcal{A}_{\mathrm{L} 2}^{5]} & \mathcal{A}_{\mathrm{L} 1}^{[5]} & \mathcal{A}_{\mathrm{M}}^{[5]} & \mathcal{A}_{\mathrm{R}]}^{[5]} & \mathcal{A}_{\mathrm{R} 2}^{[5]} & \ldots & 0 \\
0 & \mathcal{A}_{\mathrm{L} 2}^{[6]} & \mathcal{A}_{\mathrm{L} 1}^{[6]} & \mathcal{A}_{\mathrm{M}}^{[6]} & \mathcal{A}_{\mathrm{R} 1}^{[6]} & \mathcal{A}_{\mathrm{R} 2}^{[6]} & \ldots \\
0 & 0 & \mathcal{A}_{\mathrm{L} 2}^{[7]} & \ddots & \ddots & \ddots & \ddots \\
0 & 0 & 0 & \ddots & \ddots & \ddots & \ddots \\
0 & 0 & 0 & 0 & \mathcal{A}_{\mathrm{L} 2}^{[n-2]} & \ldots & \mathcal{A}_{\mathrm{M}}^{[n-2]}
\end{array}\right],
$$

with $x_{k}$ given by (14). As in [1], the parametrization $A(x) x$ of $f(x)$ is not unique.

\section{Simulation Results for 1D Advection}

Figure 1 uses $n=44$ cells with $x_{k} \in \mathbb{R}^{400}$. The width $\Delta r_{j}$ of cell $j$ and time step $t_{\mathrm{s}}(k)$ in (12) are varied to ensure stability. The initial states are determined by an empirical model relating the neutral densities and temperature to the integrated solar flux approximation $\left(F_{10.7}\right)$ and activity level $(A p)$ [5]. Hence, initial conditions such as initial neutral and ion densities are varied through $F_{10.7}$.

The states of the truth model $x_{0}$ are initialized with $F_{10.7}=210$, which is used throughout the simulation. We assume that noise-free measurements of the number density of electron and the ion velocity in 40th cell are available so that

$$
y_{k}=C\left(x_{k}\right) x_{k},
$$

where $C\left(x_{k}\right) \in \mathbb{R}^{2 \times 400}$ has entries 0 's, 1 's and $\frac{\exp \left(U_{l_{0}+l}(k)\right)}{U_{l_{0}+l}(k)}$ in which $l_{0}=390$, $l=1, \ldots, 9$. Next, we assume the initial state $x_{0}$ is unknown and estimate $x_{k}$ 


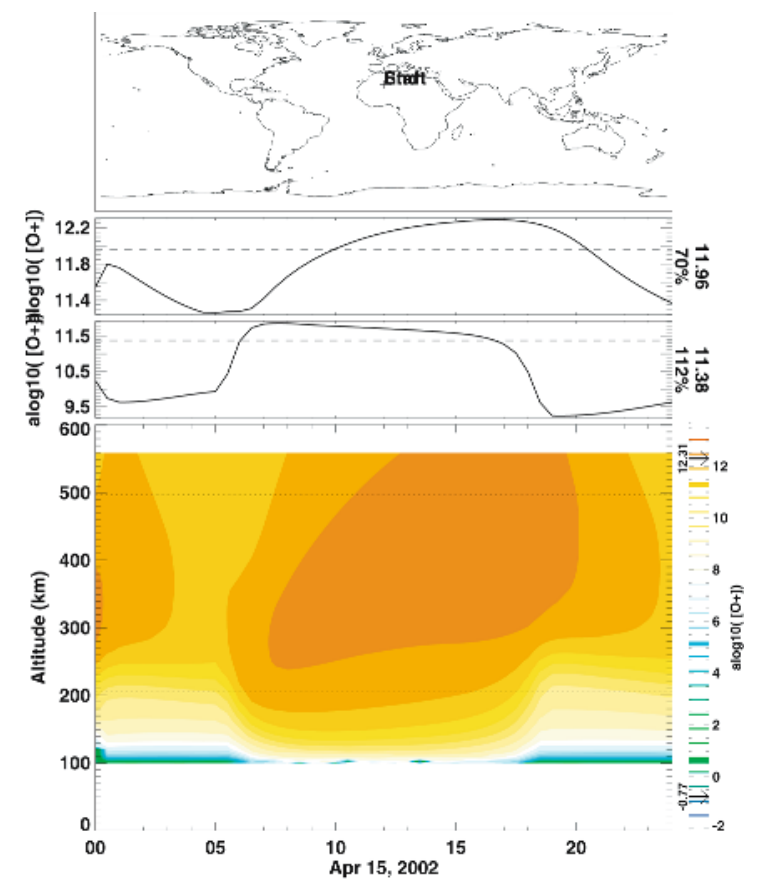

Fig. 2. Distribution of $\mathrm{O}^{+}$ions at altitudes above the location marked by ' + ' on the map (top plot). The second plot shows the log of the number density of $\mathrm{O}^{+}$along the upper dotted line in the bottom plot (around $500 \mathrm{~km}$ altitude), while the third plot shows the $\log$ of the number density of $\mathrm{O}^{+}$along the lower dotted line in the bottom plot (around $200 \mathrm{~km}$ altitude). The bottom plot shows the log of the number density of $\mathrm{O}^{+}$as a function of time and altitude. All logs are base 10.

using $y_{k}$. To do this, the estimator state is initialized with $F_{10.7}=200$ so that $x_{0}^{\mathrm{f}} \neq x_{0}$. The estimates $\hat{x}_{k}$ are obtained by using the SDRE filter, where $P_{k}$ is propagated using $P_{0}=I_{400}, Q_{k}=I_{400}, R_{k}=I_{2}, C_{k}=C\left(x_{k}^{\mathrm{f}}\right)$ and $A_{k}=A\left(x_{k}^{\mathrm{f}}\right)$; $A\left(x_{k}^{\mathrm{f}}\right)$ is given by (17) with $x_{k}$ replaced by $x_{k}^{\mathrm{f}}$. Although we set $F_{10.7}=200$ at $k=0$ to initialize the estimator, we set $F_{10.7}=210$ for all $k>0$.

Figure 2 shows the distribution of $O^{+}$at altitudes above the location marked by ' + ', obtained by using the truth model initialized with $F_{10.7}=210$. The number density and ion velocity of $\mathrm{O}^{+}$at cells 30 and 40 of the truth model are shown in Figure 3. The estimates of ion density and ion velocity obtained from the SDRE filter by using measurements from cell 40 are also shown in the same figures. Finally, estimates of the ion density and ion velocity of $O^{+}$obtained when no data assimilation is performed are also shown. Since the initial condition of the estimator $x_{0}^{\mathrm{f}}$ differs from the initial condition of the truth model $x_{0}$, the number density and the ion velocity when no data assimilation is performed differs from the number density and the ion velocity of the truth model. However, when measurements are used for data assimilation, the SDRE-based filter provides good estimates of the states of the truth model. 

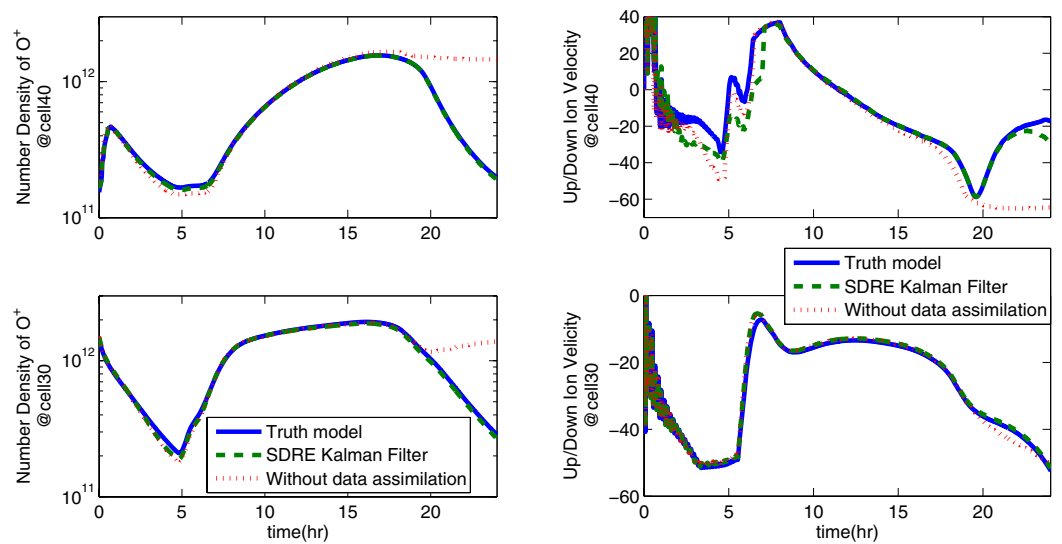

Fig. 3. Number density and ion velocity of $\mathrm{O}^{+}$at cells 30 and 40 are indicated by the solid lines. The estimates obtained when no data assimilation is performed are indicated by the dotted lines. These estimates differ from the state of the truth model because of the different initial conditions. The dashed lines indicated the SDRE filter estimates obtained when measurements from cell 40 are used for data assimilation. Note that after transients due to the erroneous initial conditions, the SDRE filter asymptotically achieves correct state estimates.

\section{References}

1. J. Chandrasekar, D. S. Bernstein, and A. Ridley. SDRE and EKF-based State Estimation for Two-Dimensional MHD Flow. 2006. preprint.

2. J. Chandrasekar, A. J. Ridley, and D. S. Bernstein. An SDRE-Based Asymptotic Observer for Nonlinear Discrete-Time Systems. In Proc. Amer. Contr. Conf., pages 3630-3635, Portland, OR, June 2005.

3. G. Evensen. Advanced Data Assimilation for Strongly Nonlinear Dynamics. Monthly Weather Rev., 125:1342-1354, 1997.

4. S. Gillijns, O. Barrero Mendoza, J. Chandrasekar, B. L. R. De Moor, D. S. Bernstein, and A. Ridley. What Is the Ensemble Kalman Filter and How Well Does it Work? In Proc. Amer. Contr. Conf., Minneapolis, MN, June 2006.

5. A. J. Ridley, Y. Deng, and G. Toth. The Global Ionosphere-Thermosphere Model (GITM). J. Atmos. Solar-Terrestr. Phys., 2006. to appear.

6. R.G. Roble, E.C. Ridley, and R.E. Dickinson. On the Global Mean Structure of the Thermosphere. J. Geophys. Res., 92:8745, 1987.

7. R.W. Schunk and A.F. Nagy. Ionospheres. Cambridge Press, Cambridge University, 2000 . 